\title{
Therapeutic Efficacy and Safety of Wavelength-Converted 660-nm 0-Switched Ruby-Like Versatile YAG Treatment on Various Skin Pigmentation Disorders
}

\author{
Boncheol Leo Goo' \\ Jin-Soo Kang ${ }^{2}$ \\ Sung Bin $\mathrm{Cho}^{2,3}$
}

\author{
${ }^{1}$ Clinique L Dermatology, Goyang, Korea \\ ${ }^{2}$ Kangskin Clinic, Seoul, Korea \\ ${ }^{3}$ Department of Dermatology and Cutaneous \\ Biology Research Institute, Yonsei University \\ College of Medicine, Seoul, Korea
}

Received July 7, 2014

Revised July 14, 2014

Accepted July 14, 2014

\footnotetext{
Correspondence

Sung Bin Cho

Department of Dermatology and Cutaneous Biology Research Institute, Yonsei University College of Medicine, 50 Yonsei-ro, Seodaemoongu, Seoul 120-752, Korea

Tel: +82-2-2228-2080

Fax: +82-2-393-9157

E-mail: drsbchodgmail.com

(c) Korean Society for Laser Medicine and Surgery

(c) This is an open access article distributed under the terms of the Creative Commons Attribution NonCommercial License (http://creativecommons.org/ licenses/by-nc/3.0) which permits unrestricted noncommercial use, distribution, and reproduction in any medium, provided the original work is properly cited.
}

\section{Background and Objectives}

Using a handpiece equipped with solid dye, 532-nm Q-switched (QS) neodymium-doped yttrium aluminum garnet (Nd:YAG) laser energy can be converted to $660-\mathrm{nm}$ laser energy for use in ruby-like versatile YAG (RuVY) laser treatment. The objective of this study is to evaluate the clinical efficacy and safety of RuVY treatment using a QS Nd:YAG laser in treatment of various cutaneous pigmentation disorders in Asian patients.

\section{Materials and Methods}

We retrospectively analyzed outcomes in 20 Korean patients who underwent wavelength-converted 660-nm RuVY treatment using a QS $\mathrm{Nd}$ :YAG laser for treatment of a variety of pigmentation disorders. RuVY treatment was performed with the settings of $660-\mathrm{nm}$ wavelength, a pulse

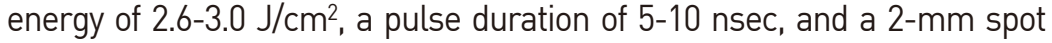
size.

\section{Results}

Among the 20 subjects included in this study, the mean number of RuVY treatments performed was $1.3 \pm 0.5$, the mean global aesthetic improvement scale (GAIS) score was $2.1 \pm 1.1$, and the patients' mean degree of satisfaction was $2.0 \pm 1.0$. Sixteen $(80 \%)$ patients also received QS low-fluenced 1,064-nm Nd:YAG laser treatment while undergoing RuVY treatment, with or without a long-pulsed 755-nm alexandrite laser. They were treated with a mean of $6.9 \pm 2.5$ sessions of QS low-fluenced 1,064-nm Nd:YAG laser treatment for a mean GAIS score of $1.9 \pm 1.1$. Eight patients were treated with long-pulsed 755-nm alexandrite laser treatment over a mean number of $1.1 \pm 0.4$ sessions, showing a mean GAIS score of $1.8 \pm 1.5$.

\section{Conclusion}

Our data suggest that RuVY treatment utilizing wavelength-converted $660-\mathrm{nm}$ laser energy is effective in treatment of various epidermal pigmentation lesions.

\section{Key words}

Nd:YAG laser; Q-switched; Ruby laser; 660-nm; Lentigines; Freckle 


\section{INTRODUCTION}

By targeting pigments via non-ablative selective photothermolysis, Q-switched (QS) lasers are widely used to effectively treat a variety of cutaneous pigmentation lesions. 'Melanin absorption is higher in QS 532-nm neodymium-doped yttrium aluminum garnet (Nd:YAG) laser treatment than other QS laser treatments; however, better absorption rates to target tissues are not always associated with better clinical outcomes. For example, higher absorption by hemoglobin of QS $\mathrm{Nd}$ :YAG laser energy at $532 \mathrm{~nm}$ significantly increases the risk of unexpected side effects, in particular damage to superficial vessels. ${ }^{2}$ Additionally, Asian patients of darker skin type with higher amounts of melanin are at higher risk of post-laser therapy hyperpigmentation and erythema. ${ }^{1,2}$

Although QS 532- and 1,064-nm Nd:YAG lasers are still the most widely used devices for treating various pigmentation disorders, QS ruby lasers with a wavelength of $694 \mathrm{~nm}$ have been shown to facilitate better absorption by melanin than QS 1,064-nm Nd:YAG lasers and weaker absorption by hemoglobin than $532-n m$ lasers. $^{3-5}$ Reportedly, QS ruby lasers have proven successful in tattoo removal and in treating cutaneous pigmentations lesions, including melasma, freckles, lentigines, café-aulait macules, Becker's nevus, and nevus of Ota. ${ }^{3,5}$ Using a handpiece equipped with solid dye, 532-nm QS Nd:YAG laser energy can be converted to 660-nm laser energy for use in ruby-like versatile YAG (RuVY) laser treatment. In the present study, we evaluated the clinical efficacy and safety of RuVY treatment using QS Nd:YAG lasers in treating various cutaneous pigmentation disorders in Asian patients.

\section{MATERIALS AND METHODS}

\section{Patients}

Twenty Korean patients lone man and 19 women with the mean age of $35.0 \pm 13.3$ years, ranging from 11 to 59 years) with Fitzpatrick skin type III-IV who previously underwent wavelength-converted 660-nm RuVY treatment using QS Nd:YAG lasers to treat pigmentation disorders were retrospectively analyzed in this study. Table 1 summarizes their demographics. The patients received RuVY treatment alone or combined treatment with other laser therapies, including QS low-fluenced 1,064-nm Nd:YAG laser treatment and long-pulsed 755$\mathrm{nm}$ alexandrite laser treatment. Among the subjects of this study, clinical diagnoses of lentigines $(N=10)$, seborrheic keratosis $(N=4)$, facial lentiginosis $(N=3)$, café-au-lait macules $(N=2)$, and freckles $(N=1)$ were noted. Also, among the 10 patients with lentigines, two were additionally diagnosed with melasma.

\section{Laser treatment}

All enrolled subjects were treated with one or two sessions of RuVY treatment using a QS Nd:YAG laser device (SPECTRA XT ${ }^{T M}$, Lutronic Corporation, Goyang, Korea). A handpiece equipped with solid dye was used to convert 532-nm wavelength laser energy to 660 $\mathrm{nm}$ wavelength laser energy. At each RuVY treatment session, a single or two passes were made with the device after administering local anesthesia with topical EMLA cream leutectic mixture of 2.5\% lidocaine $\mathrm{HCl}$ and $2.5 \%$ prilocaine; AstraZeneca AB, Södertälje, Sweden) using the settings of a 660-nm wavelength, a pulse energy of

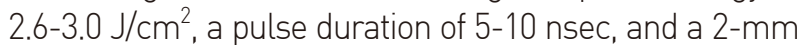
spot size at one-month intervals. In patients with both melasma and lentigines, RuVY treatment was delivered only to the lentigines, but not the melasma lesions.

Among the 20 participants, $16(80 \%)$ patients were also treated with QS low-fluenced 1,064-nm Nd:YAG laser treatment at one or two weeks after the RuVY or long-pulsed 755-nm alexandrite treatment at one-week intervals. In each patient, the whole face was treated with a 1,064-nm QS single pulse Nd:YAG laser device (SPECTRA XT ${ }^{\top M}$ ) with the settings of $1.6 \mathrm{~J} / \mathrm{cm}^{2}$, a pulse duration of 5-10-nsec, and a 7-mm spot size. A total of 1,000 to 1,500 shots were delivered on the entire face. Then, additional 1,064-nm Nd:YAG laser treatments were delivered on the pigmented lesions with the laser settings of a quick pulse-to-pulse (Q-PTP) mode, $5.0-6.0 \mathrm{~J} / \mathrm{cm}^{2}$ lirradiated at dual pulses of $2.5-3.0 \mathrm{~J} / \mathrm{cm}^{2}$ and $80-\mu \mathrm{sec}$ intervals), a pulse duration of $5-10-n s e c$, a $4-\mathrm{mm}$ spot size, and five shots per each lesion.

Eight (40\%) of the 20 patients also underwent combined treatment with a long-pulsed 755-nm alexandrite laser device (GentleMax ${ }^{\mathrm{TM}}$; Candela, Wayland, MA, USA), with the laser settings of 3 msec-pulse width, $35 \mathrm{~J} / \mathrm{cm}^{2}$, and a $6 \mathrm{~mm}$-spot size over a single pass, and a dynamic cooling device, with the settings of 0/0/20 (pre-irradiation cooling/ delay/post-irradiation cooling) msec. Immediately after the RuVY, QS low-fluenced 1,064-nm Nd:YAG laser, and long-pulsed 755-nm alexandrite laser treatments, the lesions were cooled with icepacks. The patients were recommended to apply a broad-spectrum sunscreen and avoid excessive sun exposure during the course of the laser treatments. 


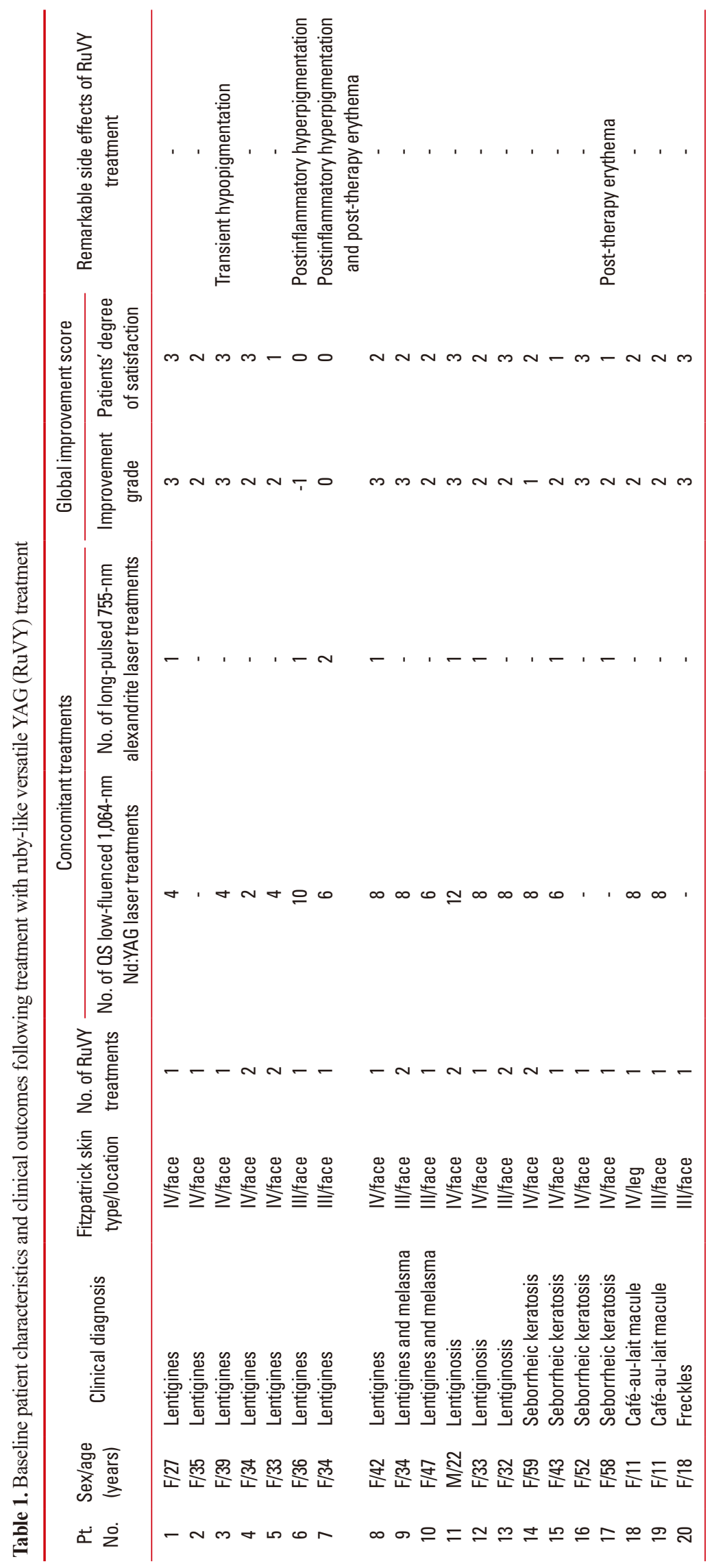




\section{Objective clinical assessment}

At baseline and one month after the final treatment, photographs were taken under normal and ultraviolet light exposures with an imaging tool (Janus ${ }^{\circledR}$, PSI corporation, Ltd., Seoul, Korea). Thereafter, two separate blinded dermatologists were asked to objectively evaluate clinical improvement in the pigmentation lesions by analyzing photographs according to the global aesthetic improvement scale (GAIS), scoring them as "worse than before" (score: -1), "clinically unchanged" (score: 0), "slightly improved" (score: 1), "moderately improved" (score: 2), and "markedly improved" (score: 3). Additionally, patients were asked to subjectively report their satisfaction with the treatment course as "unsatisfied" (score: 0), "slightly satisfied" (score: 1), "satisfied" (score: 2), and "very satisfied" (score: 3), as well as any side effects.

\section{RESULTS}

Among the 20 subjects included in this study, the mean number of RuVY treatment sessions was $1.3 \pm$ 0.5 , the mean GAIS score was $2.1 \pm 1.1$, and the patients' mean degree of satisfaction was $2.0 \pm 1.0$. As well, $16(80 \%)$ patients who also received QS low-fluenced 1,064-nm Nd:YAG laser treatment during the course of RuVY treatment, with or without a long-pulsed 755-nm alexandrite laser. They underwent $6.9 \pm 2.5$ sessions of QS low-fluenced 1,064-nm Nd:YAG laser treatment for a mean GAIS score of $1.9 \pm 1.1$; the patients' degree of satisfaction with this treatment course was $1.9 \pm 1.0$. Eight patients were treated over a mean $1.1 \pm 0.4$ sessions of long-pulsed 755-nm alexandrite laser treatment for a mean GAIS score of $1.8 \pm 1.5$ and a patient degree of satisfaction of $1.5 \pm 1.2$. Among them, four patients (cases 7, 12, 15, and 17) demonstrated unsatisfactory clinical responses to the long-pulsed alexandrite laser treatment, and therefore, RuVY treatment was added for better treatment outcomes. Meanwhile, despite remarkable clinical improvement, four patients (cases 1 , $6,8,11$ ) first treated with a long-pulsed alexandrite laser device underwent follow-up RuVY treatment for residual pigmented lesions. Three patients were treated with RuV alone, presenting both a mean GAIS score and a degree of satisfaction of $2.7 \pm 0.6$.

In cases of single or multiple lentigines (cases 1-10),
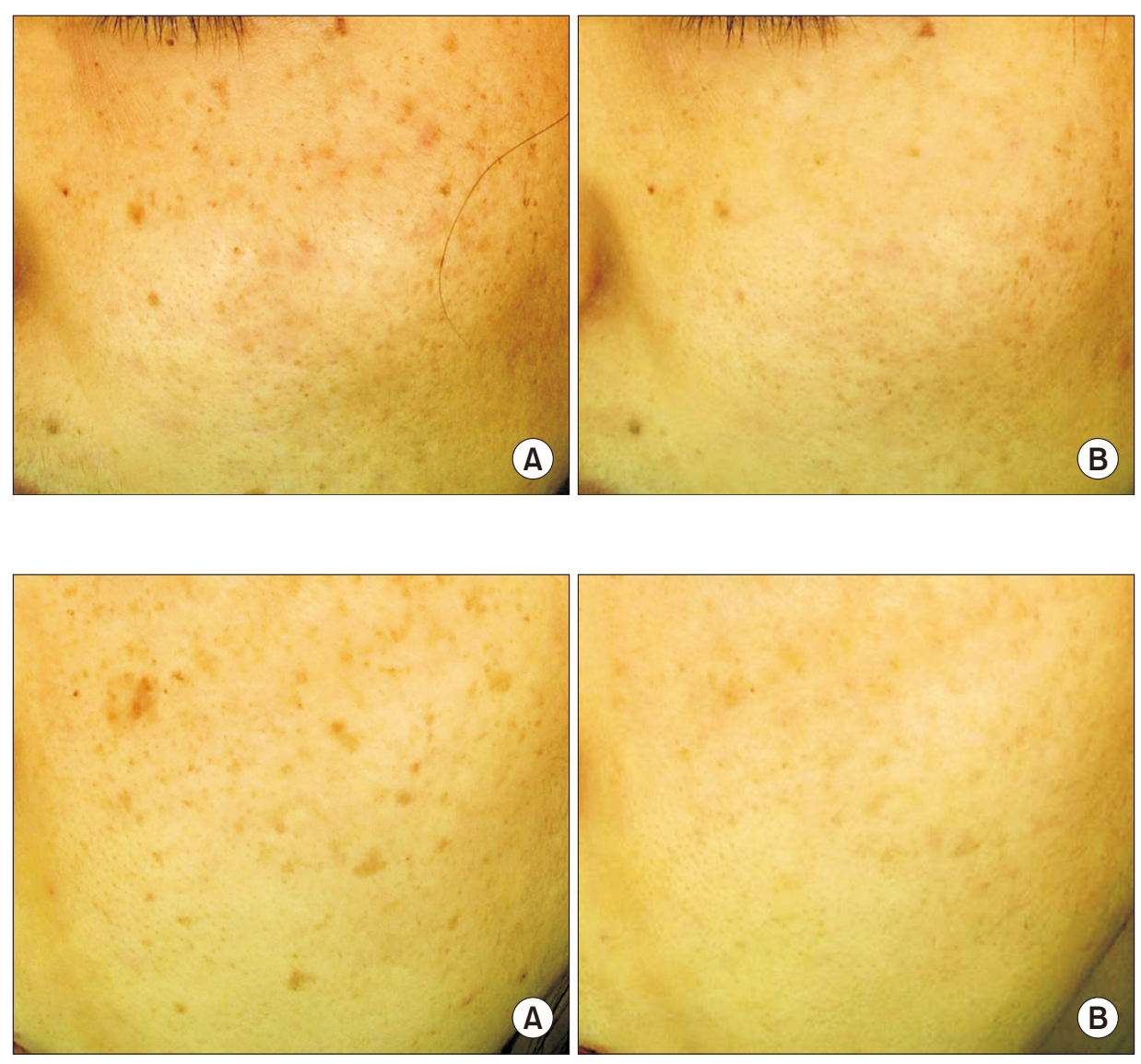

Fig. 1. Case 3. A 39-year-old female patient with multiple facial lentigines (A) before and (B) one month after one session of RuVY treatment and four sessions of Q-switched (QS) $\mathrm{Nd}$ :YAG laser treatment at low fluence. Transient hypopigmentation, which developed after the RuVY treatment, was also improved.

Fig. 2. Case 9. A 34-year-old female patient with multiple facial lentigines (A) before and (B) one month after two sessions of RuVY treatment and eight sessions of Q-switched (QS) $\mathrm{Nd}$ :YAG laser treatment at low fluence. 
the mean GAIS score was $1.9 \pm 1.4$ and the patients degree of satisfaction was $1.8 \pm 1.1$ (Fig. 1, 2; case 3, 9). Among the 10 patients with lentigines, two patients with Fitzpatrick skin type III developed postinflammatory hyperpigmentation after the RuVY treatment. One patient (case 6) presented with lentiginous lesions along the periorbital areas and exhibited partial clinical improvement after long-pulsed alexandrite laser treatment; however, the patient developed postinflammatory hyperpigmentation after RuVY treatment of residual pigmented lesions (Fig. 3). The other patient (case 7), who presented with coin-sized light brown-colored lentigines along the lateral cheeks, was refractory to the long-pulsed alexandrite laser treatment; however, the patient developed postinflammatory hyperpigmentation and prolonged erythema after undergoing RuW treatment. Transient hypopigmentation was observed in one patient with lentigines (case 3), which was markedly improved after four sessions of QS 1,064$\mathrm{nm}$ Nd:YAG laser treatment at low fluence (Fig. 1).

In patients with facial lentiginosis (cases 11, 12, 13), a long-pulsed alexandrite laser device was effectively used to treat the dark brown-colored skin lesions (case 11; Fig. 4). However, small-sized light brown lesions, as well
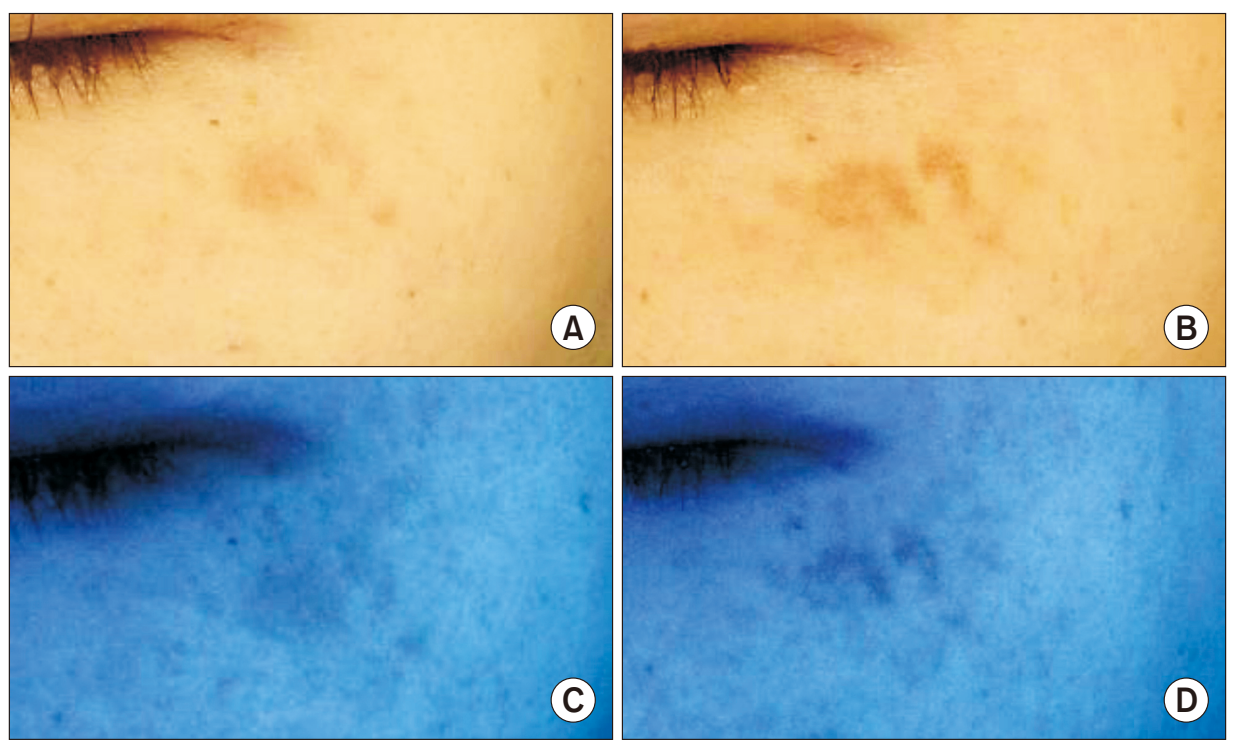

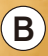

Fig. 3. Case 6. A 36-year-old female patient with RuVY treatment-induced postinflammatory hyperpigmentation along the periorbital areas (A, C) before and $(\mathrm{B}, \mathrm{D})$ one month after RuVY treatment. Photos were taken under (A, B) normal light exposure and $(\mathrm{C}, \mathrm{D})$ ultraviolet light exposure.
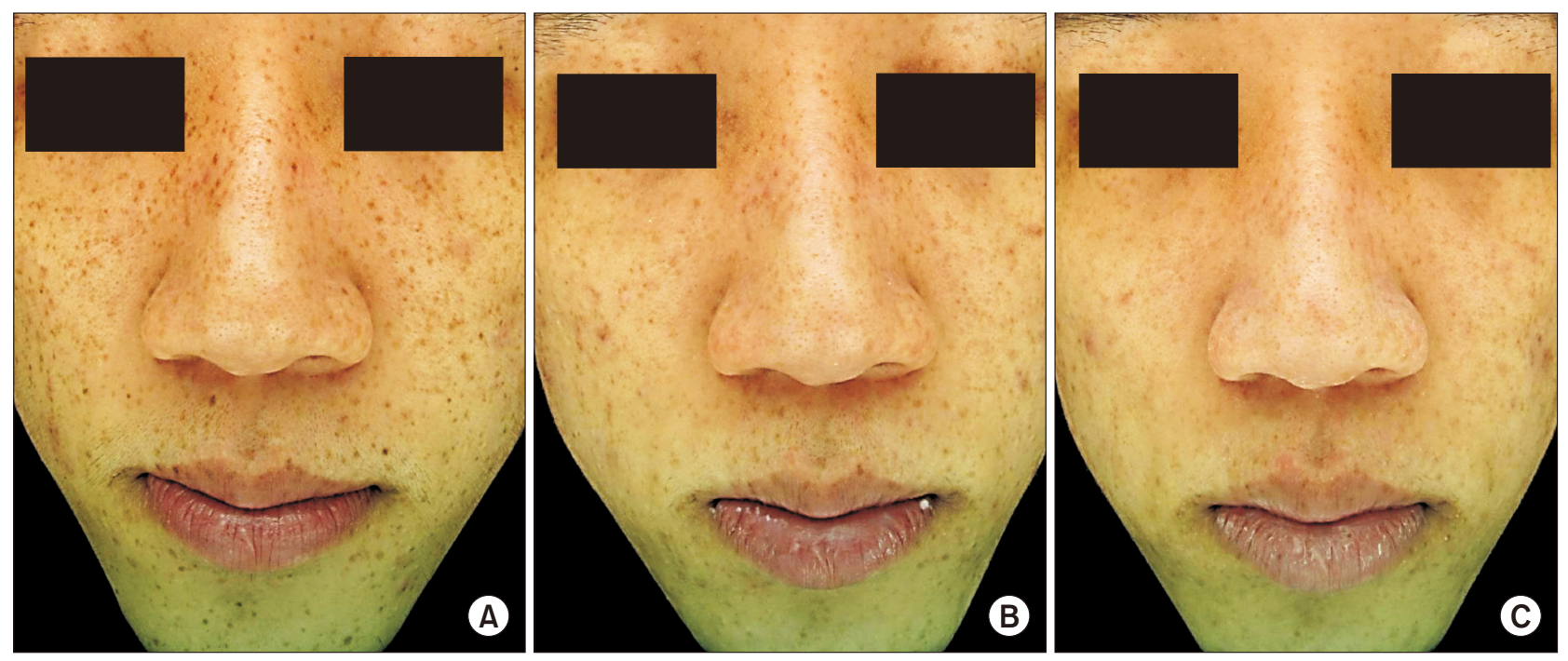

Fig. 4. Case 11. A 22-year-old male patient with facial lentiginosis. (A) Before, (B) one week after one session of long-pulsed 755-nm alexandrite laser and four sessions of QS low-fluenced Nd:YAG laser treatment, and (C) one month after an additional two sessions of RuVY treatment and eight sessions of QS low-fluenced Nd:YAG laser treatment. 

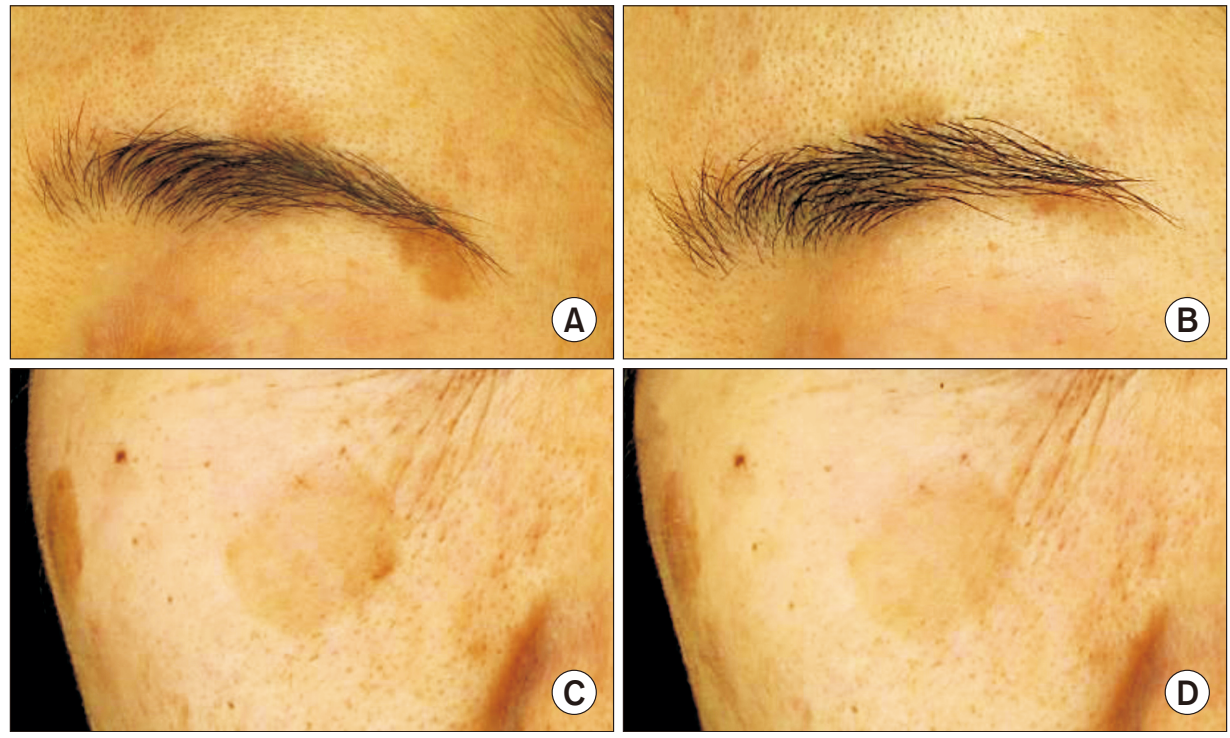

Fig. 5. Case 16. A 52-year-old female patient with seborrheic keratosis. (A) Before and (B) one month after one session of RuVY treatment. Case 14. A 59-year-old female patient with seborrheic keratosis. (C) Before and (D) one month after two sessions of RuVY treatment and eight sessions of QS low-fluenced Nd:YAG laser treatment. as upper eyelid lesions, demonstrated better therapeutic response to RuVY treatment followed by QS low-fluenced 1,064-nm Nd:YAG laser treatments without remarkable side effects. As well, darkly pigmented seborrheic keratosis lesions also responded well to the RuVY treatment (Fig. 5A, B); however, RuVY treatment of thick and light brown colored seborrheic keratosis resulted in unsatisfactory therapeutic outcomes, even with repetitive treatment (Fig. 5C, D). One patient with freckles (case 20) presented with near total to complete improvement thereof with a single session of RuVY treatment without remarkable side effects.

\section{DISCUSSION}

In this study, we attempted to demonstrate the clinical efficacy and safety of RuVY treatment on various epidermal pigmented lesions, including lentigines, facial lentiginosis, seborrheic keratoses, café-au-lait macules, and freckles. RuVY treatment was performed by converting 532nm QS Nd:YAG laser energy to 660-nm laser energy. Although QS lasers are preferred and widely used for the treatment of epidermal and dermal pigmented disorders, a shorter pulse duration can result in post-therapy hyperor hypopigmentation due to higher risk of unwanted photomechanical effects on adjacent tissue components, especially blood vessels. ${ }^{6}$ As with QS ruby lasers with a wavelength of 694-nm, ${ }^{3-5}$ the wavelength-converted $660-n m$ laser, which is a quite similar but not identical to 694-nm lasers, was theoretically expected to offer better absorption by melanin and weaker absorption by hemoglobin.
The efficacy and safety of QS 694-nm ruby lasers for solar lentigines in patients with Fitzpatrick skin type II-IV has been evaluated once previously. ${ }^{7}$ Therein, the authors utilized a QS ruby laser device with the laser settings of

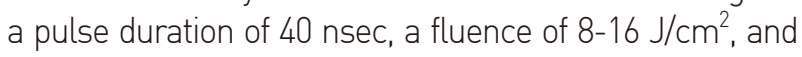
a 3-mm spot size, and reported the complete removal all of solar lentigines after a single or multiple sessions of QS ruby laser treatment. ${ }^{7}$ In the present study, we treated lentigines with the laser settings of a converted wavelength of $660 \mathrm{~nm}$, a pulse duration of 5-10 nsec, a fluence of 2.6-3.0 J/ $\mathrm{cm}^{2}$, a 2-mm spot size, and a mean 1.3 \pm 0.5 sessions, for a mean GAIS of $1.9 \pm 1.4$. We suspect that differences in the wavelengths, as well as the laser settings, especially the shorter pulse duration and lower pulse energy, could have affected to the clinical outcomes.

Additionally, postinflammatory hyper- or hypopigmentation after the QS 694-nm ruby laser treatment on lentigines was reportedly observed in $7.8 \%$ of the patients with Fitzpatrick skin type II, in $9.8 \%$ with type III, and in $16.6 \%$ with type IV in the aforementioned previous study. ${ }^{7}$ In the present study, we treated seven patients with Fitzpatrick skin type III and 13 patients with type IV by RuVY treatment. Upon doing so, two $(10 \%)$ of 20 participants showed postinflammatory hyperpigmentation (all were Fitzpatrick skin type III); two (10\%) patients experienced prolonged post-therapy erythema (all were type III); and one (5\%) patient showed transient hypopigmentation (a patient with type IV). Accordingly, based on results reported here and the literature, any risk of postinflammatory dyspigmentation and prolonged erythema after the RuVY treatment seems to be associated with Fitzpatrick skin type, pulse energy, 
and concomitant 1,064-nm low-fluenced Nd:YAG laser treatments.

Pigment-targeting lasers with longer pulse duration have been used for the treatment of various pigmented lesions in the epidermis, including congenital melanocytic nevus, lentigines, and seborrheic keratoses. ${ }^{8,9}$ Our study group also reported that 755-nm alexandrite laser treatments with the laser settings of a high fluence and long pulse duration (3-msec) results in high clearance rates on the various types and colors of seborrheic keratoses with low risk of side effects. ${ }^{9}$ Pigment-specific long-pulsed lasers can be effectively used in highfluenced laser treatment to eliminate large nests of nevi cells, whereas QS pigment-specific lasers are used to target individual pigments, as in our patients with seborrheic keratoses. ${ }^{10}$ However, in a previous study, about $40 \%$ of Asian patients reportedly experienced postinflammatory hyperpigmentation after undergoing long-pulsed alexandrite laser treatment using a large beam size, which was suggested to deliver the laser energies on the adjacent vascular structures, to remove epidermal pigmented lesions. ${ }^{11}$

As RuVY treatment involves delivery of laser energy over a small spot size of 2-3 $\mathrm{mm}$ and has a wavelength of $660 \mathrm{~nm}$ for relatively weaker absorption rate by hemoglobin, the risk of vascular damage and associated postinflammatory dyspigmentation is potentially low. However, treatment of large pigmented lesions using a small spot sized typically necessitates a longer procedure time and additional effort to irradiate the lesions. Therefore, we suggest the use of long-pulsed pigmentspecific lasers for treatment of large nests of pigmented lesions and RuVY treatment for light brown to black macular skin lesions of smaller sizes.

In conclusion, our data suggest that RuVY treatment, utilizing a converted wavelength of $660 \mathrm{~nm}$, can be effectively used for the treatment of various epidermal pigmented lesions. However, further investigations are needed to confirm our findings and to compare different QS lasers in optimized and prospective studies.

\section{REFERENCES}

1. Chan HH, Fung WK, Ying SY, Kono T. An in vivo trial comparing the use of different types of $532 \mathrm{~nm} \mathrm{Nd:YAG} \mathrm{lasers} \mathrm{in} \mathrm{the}$ treatment of facial lentigines in Oriental patients. Dermatol Surg 2000;26:743-9.

2. Anderson RR, Margolis RJ, Watenabe S, Flotte T, Hruza GJ, Dover JS. Selective photothermolysis of cutaneous pigmentation by Q-switched Nd: YAG laser pulses at 1064, 532, and $355 \mathrm{~nm}$. J Invest Dermatol 1989;93:28-32.

3. Tse Y, Levine VJ, McClain SA, Ashinoff R. The removal of cutaneous pigmented lesions with the $\mathrm{Q}$-switched ruby laser and the Q-switched neodymium: yttrium-aluminum-garnet laser. A comparative study. J Dermatol Surg Oncol 1994;20:795800.

4. Chan NP, Ho SG, Shek SY, Yeung CK, Chan HH. A case series of facial depigmentation associated with low fluence $Q$-switched 1,064 nm Nd:YAG laser for skin rejuvenation and melasma. Lasers Surg Med 2010;42:712-9.

5. Jang WS, Lee CK, Kim BJ, Kim MN. Efficacy of 694-nm $\mathrm{Q}$-switched ruby fractional laser treatment of melasma in female Korean patients. Dermatol Surg 2011;37:1133-40.

6. Ara G, Anderson RR, Mandel KG, Ottesen M, Oseroff AR. Irradiation of pigmented melanoma cells with high intensity pulsed radiation generates acoustic waves and kills cells. Lasers Surg Med 1990;10:52-9.

7. Sadighha A, Saatee S, Muhaghegh-Zahed G. Efficacy and adverse effects of $\mathrm{Q}$-switched ruby laser on solar lentigines: a prospective study of 91 patients with Fitzpatrick skin type II, III, and IV. Dermatol Surg 2008;34:1465-8.

8. Trafeli JP, Kwan JM, Meehan KJ, Domankevitz Y, Gilbert S, Malomo K, et al. Use of a long-pulse alexandrite laser in the treatment of superficial pigmented lesions. Dermatol Surg 2007;33:1477-82.

9. Kim YK, Kim DY, Lee SJ, Chung WS, Cho SB. Therapeutic efficacy of long-pulsed 755-nm alexandrite laser for seborrheic keratoses. J Eur Acad Dermatol Venereol 2013. [Epub ahead of print]

10. Ueda S, Imayama S. Normal-mode ruby laser for treating congenital nevi. Arch Dermatol 1997;133:355-9.

11. Ho SG, Yeung CK, Chan NP, Shek SY, Chan HH. A comparison of $Q$-switched and long-pulsed alexandrite laser for the treatment of freckles and lentigines in oriental patients. Lasers Surg Med 2011;43:108-13. 